\title{
Repellent Signaling by Slit Requires the Leucine-Rich Repeats
}

\author{
Robin Battye, Adrienne Stevens, Robert L. Perry, and J. Roger Jacobs \\ Department of Biology, McMaster University, Hamilton Ontario, L8S 4K1 Canada
}

Slit is a repellent axon guidance cue produced by the midline glia in Drosophila that is required to regulate the formation of contralateral projections and the lateral position of longitudinal tracts. Four sequence motifs comprise the structure of Slit: a leucine-rich repeat (LRR), epidermal growth factor-like (EGF) repeats, a laminin-like globular (G)-domain, and a cysteine domain. Here we demonstrate that the LRR is required for repellent signaling and in vitro binding to Robo. Repellent signaling by slit is reduced by point mutations that encode single amino acid changes in the LRR domain. By contrast to the EGF

Growing axons navigate through the developing nervous system by integrating varied adhesive, attractive, and repellent signals, delivered by cell contact or diffusion through the extracellular matrix (ECM) (Tessier-Lavigne and Goodman, 1996). The midline of the CNS is an important source of guidance signals. Most interneurons cross once and avoid the midline thereafter. At least two signals regulate axon guidance at the midline of all nervous systems examined to date. The Netrin pathway communicates an attractive cue from midline cells to enable midline axon crossing (Kennedy et al., 1994). The Slit pathway communicates a repellent cue from midline cells to restrict axon crossing (Battye et al., 1999; Kidd et al., 1999; Yuan et al., 1999; Zhou et al., 2000).

Midline guidance signals Netrin and Slit are both produced by the midline glia (MG) in Drosophila (for review, see Jacobs, 2000). Slit, a secreted glycoprotein, is found on the surface of the MG and at low levels on the surface of all CNS axons (Rothberg et al., 1988; Rothberg et al., 1990.; Kidd et al., 1999). Slit binds to three Roundabout receptors (Robo, Robo2, and Robo3) on axons and growth cones to inhibit filopodial extension and growth toward the midline and to specify the lateral position of axons in the longitudinal tracts (Brose et al., 1999; Li et al., 1999; Murray and Whitington, 1999; Rajagopalan et al., 2000; Simpson et al., 2000a,b).

The functions of the structural domains of Slit are unknown. Four leucine-rich repeats (LRRs) comprise most of the protein. This motif is found in matrix proteins that bind to collagen or laminin (Matsushima et al., 2000), in transmembrane receptors, and in cell surface adhesion molecules (Gay et al., 1991; Nose et al., 1992; Yamagata et al., 1994). LRRs are thought to be required

Received Jan. 4, 2001; revised March 23, 2001; accepted March 23, 2001.

This work was supported by the Canadian Institutes for Health Research. We are grateful to Kim Bland for providing a robo expression vector, to Guy Tear for sending robo mutants and antibody, and to Corey Goodman for forwarding many alleles of slit and providing mAb 1D4. Spyros Artavanis-Tsakonas provided the Slit hybridoma cells.

Correspondence should be addressed to J. Roger Jacobs, Department of Biology, McMaster University, 1280 Main Street W., Hamilton Ontario, L8S 4K1 Canada. E-mail: jacobsr@mcmaster.ca.

Copyright (C) 2001 Society for Neuroscience $\quad 0270-6474 / 01 / 214290-09 \$ 15.00 / 0$ or G-domains, the LRR domain is required in transgenes to affect axon guidance. Finally, we show that the midline repellent receptor, Robo, binds Slit proteins with internal deletions that also retain repellent activity. However, Robo does not bind Slit protein missing the LRR. Taken together, our data demonstrate that Robo binding and repellent signaling by Slit require the LRR region.

Key words: axon guidance; Drosophila; midline glia; Slit; repulsion; Roundabout

for protein recognition and adhesion events (Hocking et al., 1998).

Drosophila Slit also contains seven EGF repeats, similar in sequence to the Notch EGF repeats. These repeats also are implicated in protein recognition and ligand binding events (Lieber et al., 1992). The C-terminal region of Slit contains a laminin-like globular (G)-domain, also termed an ALPS (for agrin, laminin, perlecan, slit) domain (Rothberg and ArtavanisTsakonas, 1992). The agrin G-domain is required for nerve-tomuscle signaling that triggers formation of the neuromuscular synapse (Cornish et al., 1999).

Slit can be proteolytically cleaved. The amino terminal fragment enhances sensory axon branching in vitro (Wang et al., 1999). Repellent activities of either fragment have not been determined.

Our sequence analysis of seven mutations indicates that single amino acid changes in the LRR regions and terminations of translation in the EGF repeats reduce repellent signaling by slit. We have also generated three slit transgenes, with different internal deletions, to determine which structural domains are required for repellent signaling. An intact LRR domain is required in transgenes to restore midline guidance in slit mutants and for Slit binding to the Robo receptor in vitro. Expression of transgenes lacking the EGF or G-domain restore slit function and encode proteins that bind Robo. These data indicate that the LRR domain is required for repellent signaling by Slit.

\section{MATERIALS AND METHODS}

Stocks. Most slit and robo alleles were isolated on a background deficient for fasciclin III and fasciclin I (Seeger et al., 1993). slit ${ }^{2}$ was isolated by Nüsslein-Volhard et al. (1984), slit ${ }^{\mathrm{E} 158}$ was isolated by A. Kolodkin (Johns Hopkins University), and slit ${ }^{\mathrm{F} 81}$ and slit $^{\mathrm{F} 119}$ were isolated by $\mathrm{H}$. Bellen (Rothberg et al., 1990). robo $1^{1}$ was provided by G. Tear (Seeger et al., 1993). All stocks have been restored to a common wild-type background. $D f(2 R) W M G$ was obtained from the Indiana stock center. Transgene expression was regulated by $P[$ slit1.0-GAL4] (Christian Klämbt, Münster), $P[e n-G A L 4]$ (Orihara et al., 1999), and P[elavC155GAL4] (Lin and Goodman, 1994).

Slit antibody generation. Slit hybridoma 6D.4 was donated by S. Artavanis-Tsakonas (Rothberg et al., 1988) and reestablished in RPMI Medium (Life Technologies-BRL, Gaithersburg, MD) supplemented 
with $10 \%$ fetal calf serum (Life Technologies-BRL) and penicillin streptomycin (Life Technologies-BRL). Medium from subcloned mAb 6D.4 was purified on protein-G Sepharose 4 beads (Pharmacia Biotechnology, Arlington Heights, IL). This antibody binds to a region of in vitro translated Slit between EGF-2 and EGF-6.

Immunocytochemistry. Drosophila embryo collection, fixation, and immunocytochemistry were adapted from Patel (1994). BP102, mAb 1D4, mAb Slit, mAb Robo, [provided by N. Patel (University of Chicago), C. Goodman (University of California at Berkeley), and G. Tear (King's College), respectively], and anti- $\beta$-galactosidase (gal) (Cappel, West Chester, PA) were diluted in PBS containing 0.5\% Triton X-100. Incubation in biotinylated secondary antibody (Vector Laboratories, Burlingame, CA) was followed by incubation in Vector Laboratories Elite $\mathrm{ABC}$. The first label reacted with 3,3-diaminobenzidine tetrahydrochloride (DAB; Life Technologies-BRL) in the presence of $0.03 \%$ nickel/ cobalt chloride. Second or single labels were reduced with DAB. Nerve cords were dissected or embryos were mounted whole and visualized on a Zeiss Axiophot microscope. Figures were prepared from scanned slides with Adobe Photoshop.

Viability. Viability of each slit mutant was assessed in a $y w$ background, using a $\mathrm{CyO}\left[y^{+}\right]$balancer as a late embryonic and larval marker. Embryonic lethality monitored 1000 selected embryos, plated on apple juice agar at $22^{\circ} \mathrm{C}$. After $48 \mathrm{hr}$, unhatched embryos were dissected, and counts were taken from infertile, balanced, and mutant embryos lacking pigmented mouth hooks. Larvae were selected from the same collections, based on absence of the $C y O[y+]$ balancer. Larvae were aged at $22^{\circ} \mathrm{C}$ and observed until pupation or death.

Nerve cord length. BP102-labeled homozygous slit mutants embryos were collected on the basis of lac $Z$ balancer staining and measured under camera Lucida at $25 \times$ projection.

Fluorescence microscopy. Manually devitellinized embryos were dissected on glass in PBS and fixed for $10 \mathrm{~min}$ in $4 \%$ paraformaldehyde. The dissections were washed in buffer and incubated for $30 \mathrm{~min}$ in rhodamine-labeled phalloidin (Verheyen and Cooley, 1994) (Molecular Probes R415) and mounted in glycerol with $p$-phenylene D-amine (Sigma, St. Louis, MO) as an anti-bleaching agent. Projections made from confocal images were collected with a Zeiss 310 microscope.

Genomic analysis. Several cosmids from region 52D were screened by PCR for $5^{\prime}$ and $3^{\prime}$ domains of the slit DNA (5'for 5'-ACTCGAGCGACTGGACATCT-3', 5' rev 5'-GTCGTCGAAAGCTGGAGAAC-3', 3' for 5'-GCACAGCAGGCATACAAGAA-3', and 3' rev 5'-AGCAATTGGGTAGTCGCATC $-3^{\prime}$ ). Three genomic clones (EDGP cosmids 60E2, 113E7, and 118G10) that contained both $5^{\prime}$ and $3^{\prime}$ slit cDNA were isolated and sequenced and confirmed the revised cDNA sequence (AF 126540). PCR primer pairs were designed to flank each of the 19 slit exons and tested using cosmid and wild-type genomic DNA and a stock deficient for slit, $(D f(2 R) W M G)$. Mutant genomic DNA was obtained from single homozygous embryos or larva not carrying a $C y O[y+]$ balancer in a $y w^{-}$genetic background. Individuals were washed in sterile $\mathrm{ddH}_{2} \mathrm{O}$ and transferred to a PCR tube containing $83 \mu \mathrm{l}$ of PCR-buffered sterile $\mathrm{ddH}_{2} \mathrm{O}$ in which they were crushed, and then the remaining PCR reagents were added. Pure single-banded products were purified (Qiagen 28104; Qiagen, Hilden, Germany) for each individual sample and sequenced. At least three independently sequenced products were generated for each primer pair and genetically mutant allele. Sequencing revealed 18 silent polymorphisms and an Ile/Asn (ATC/AAC) dimorphism at nucleotide 2660 .

slit $c D N A$. A new slit cDNA was constructed on the basis of fragments of clones provided by Rothberg et al. (1988) and PCR-generated bridges that were based on genomic cosmids (118G10 and 60E2). Primers were used that overlaid known restriction sites and in addition generated a $5^{\prime}$ HindIII (5'-CATAAAGCTTCCACAATGGCCGCGCC-3') and a 3' SpeI (5'-CATCGTGCGCAAGTGCGGAACTAGTAACAA-3') restriction site for subcloning into Stratagene pBS KSII+. All products were completely sequenced and verified against the amended slit cDNA and genomic sequence.

Transgenic deletion constructs. Three transgenic constructs were created that deleted specific domains of the slit cDNA shown in Figure 4. From a complete slit construct [HindIII (-8 bp) to SpeI (4461 bp)], we generated slit $\Delta L 1-L 4$ [Bpu1102I (134) to BstEII(2539) treated with mung bean nuclease (MBN;, Life Technologies-BRL)], slit $\Delta E 2-E 6$ [NsiI (2913) to MunI (3463) treated with MBN], and slit $\Delta G-E 7$ [SspBI (3733) to $S$ fiI (4231) treated with T4 DNA polymerase]. Deletion constructs engineered with a C-terminal HA epitope tag were subcloned into
$P[U A S T]$, and transformant lines were generated and mapped by standard procedures. The epitope tag was weakly immunodetectable in vivo.

Midline rescue and overexpression. Transformant lines generated from the slit deletion constructs were crossed into a slit ${ }^{2}$ background and mated with $P\left[\right.$ slit1.0-GAL4],slit ${ }^{2} / \mathrm{CyO}[\mathrm{lacZ}]$ flies. Embryos were assayed by staining with mAbs BP102 and 6D.4 and then selected by screening of the marked balancer using $\alpha-\beta$-gal. Overexpression was produced by crosses generating $P[$ slit1.0-GAL4];P[UAS-slit $]$ and similarly using $P[$ en$G A L 4]$ and $P[$ elavC155-GAL4] lines. Embryos were immunolabeled with $\mathrm{mAb} \mathrm{BP} 102$, and slit overexpression was monitored in embryos expressing transgenes with the Slit epitope to mAb 6D.4 or with antibody to the HA epitope.

In vitro binding. To determine properties of Slit and Robo binding in vitro, $20 \mu \mathrm{l}$ of biotin-translated protein from each of the slit constructs (Biotin In Vitro translation kit, Roche) was incubated with $20 \mu \mathrm{l}$ of unlabeled Robo, rotated at $4^{\circ} \mathrm{C}$ for $2 \mathrm{hr}$. After incubation, $25 \mu \mathrm{l}$ of avidin Agarose (Roche) was added to each tube and incubated for 15-20 hr rotating at $4^{\circ} \mathrm{C}$. After washing, the biotin bound to the avidin complex was eluted by rotating the Agarose with $50 \mu \mathrm{l}$ of elution buffer $(2 \mathrm{mM}$ D-Biotin-B-Hydroxysuccinimidester (Roche), 0.1 м PB, $0.15 \mathrm{M} \mathrm{NaCl}, \mathrm{pH}$ 7.2) for $1 \mathrm{hr}$ after which $10 \mu \mathrm{l}$ of $5 \times$ sample buffer was added, and each mixture was heated to $80^{\circ} \mathrm{C}$ for $5 \mathrm{~min}$. For each construct, $12 \mu \mathrm{l}$ of supernatant was loaded onto each of three $4 \%$ gels for SDS-PAGE alongside a wide range color maker and then blotted onto a polyvinylidene difluoride membrane. Each membrane was washed and blocked before being separated for incubation with $\mathrm{mAb}$ Slit, mAb Robo, or avidin-HRP. After washing, the Slit and Robo membranes were incubated with G $\alpha \mathrm{M}$ HRP, and then all membranes were washed and then reacted with $2 \mathrm{ml}$ of ECL (Amersham Pharmacia Biotech) chemoluminescence for detection of HRP-bound primary antibody or biotinylated proteins on Kodak X-OMAT AR Film.

\section{RESULTS \\ Midline axon guidance in embryos mutant for hypomorphic alleles of slit}

To identify the structural requirements for repellent signaling by slit, we have examined the midline guidance phenotype of 13 alleles of slit generated by P-element insertions and ethylmethyl sulfonate (EMS) mutagenesis (Rothberg et al., 1990; Seeger et al., 1993). These alleles present a series of reduced midline repellent phenotypes, as assessed by the frequency of midline crossing of longitudinal tract axons labeled by fasciclin II. For illustrative purposes, we have grouped the alleles on the basis of midline guidance phenotype into phenotypic severe, moderate, and mild alleles (Table 1).

Wild-type late embryonic nerve cords have a bilateral set of three fasciclin II-expressing longitudinal fascicles that maintain a constant distance from the midline (Fig. 1A). Midline separation is maintained in embryos with a single copy of the slit gene (Fig. $1 B$ ). Complete midline fusion and a loss of all intersegmental longitudinal axons are seen in embryos homozygous for a deficiency uncovering slit (Fig. 1C). All of the mutant alleles that we examined generate slit transcript, assessed with a probe complementary to the G-domain. However, slit alleles 2, GA20, and 1912 generate no detectable Slit protein, using a monoclonal antibody raised to the EGF and G-domains of Slit (Rothberg et al., 1988). These severe mutants have a similar midline fusion of axons and retain few intersegmental axon projections (Fig. $1 D, E$, arrows). Nerve cords with fewer intersegmental axons had less nerve cord condensation (shortening) during development. The wild-type nerve cord was $73 \%$ of embryo length at stage 16 , whereas a severe slit nerve cord was $90 \%$ of embryo length (Table 1).

The remaining five EMS and three insertion alleles produce immunodetectable protein. Three P-element insertion alleles $\left(s l i^{\mathrm{F} 11}, s l i^{\mathrm{F} 119}\right.$, and $\left.s l i^{\mathrm{E} 158}\right)$ label less intensely for mRNA than the EMS alleles (data not shown). These alleles generate less complete midline fusion of axons. In moderate alleles, all longitudinal 


\begin{tabular}{|c|c|c|c|c|c|c|}
\hline Allele & $\begin{array}{l}\text { First larval instar: } \\
\text { percentage of } \\
\text { expected }^{a}\end{array}$ & $\begin{array}{l}\text { Latest observed } \\
\text { stage }\end{array}$ & Nerve cord length & $\begin{array}{l}\text { Slit protein } \\
\text { detected }\end{array}$ & $\begin{array}{l}\text { Midline guidance } \\
\text { phenotype }^{b}\end{array}$ & $\begin{array}{l}\text { robo }^{1} / \text { slit: } \text { percentage } \\
\text { of segments with } \\
\text { crossovers }^{c}\end{array}$ \\
\hline $\mathrm{yw}^{-}$ & $98.0(429)$ & Adult & $0.73 \pm 0.04$ & $\mathrm{Y}$ & Normal & $0(250)$ \\
\hline \multicolumn{7}{|l|}{ EMS alleles } \\
\hline slit $^{2}$ & $1.0(978)$ & $\mathrm{L} 1$ & $0.88 \pm 0.06$ & $\mathrm{~N}$ & Severe & 45 (227) \\
\hline slit $^{\mathrm{GA} 20}$ & $1.9(956)$ & L1 & $0.90 \pm 0.02$ & $\mathrm{~N}$ & Severe & $60(219)$ \\
\hline slit $^{\mathrm{GA} 178}$ & $13(914)$ & L1 & $0.91 \pm 0.03$ & $\mathrm{Y}$ & Severe & $61(227)$ \\
\hline slit $^{\mathrm{GA9} 945}$ & $2.3(475)$ & $\mathrm{L} 1$ & $0.88 \pm 0.02$ & $\mathrm{~N}$ & Severe & $46(215)$ \\
\hline slit $^{3149}$ & $5.1(473)$ & $\mathrm{L} 2$ & $0.94 \pm 0.02$ & $\mathrm{Y}$ & Severe & $63(318)$ \\
\hline slit $^{1912}$ & $7.4(484)$ & Pupa & $0.92 \pm 0.02$ & $\mathrm{~N}$ & Moderate & $60(231)$ \\
\hline slit $^{550}$ & $0.5(924)$ & $\mathrm{L} 1$ & $0.89 \pm 0.03$ & $\mathrm{Y}$ & Moderate & $48(283)$ \\
\hline slit $^{532}$ & $7.1(971)$ & Adult & $0.76 \pm 0.05$ & $\mathrm{Y}$ & Moderate & $46(281)$ \\
\hline slit $^{2990}$ & $2.0(438)$ & Adult & $0.85 \pm 0.04$ & $\mathrm{Y}$ & Mild & 45 (267) \\
\hline \multicolumn{7}{|c|}{ P-element insertions } \\
\hline slit $^{\mathrm{F} 81}$ & $11(970)$ & Adult & $0.82 \pm 0.06$ & $\mathrm{Y}$ & Mild & $41(232)$ \\
\hline slit $^{\mathrm{F} 119}$ & $17(943)$ & Adult & $0.80 \pm 0.07$ & $\mathrm{Y}$ & Mild & $37(229)$ \\
\hline slit $^{\mathrm{E} 158}$ & $3.1(953)$ & Pupa & $0.82 \pm 0.04$ & $\mathrm{Y}$ & Mild & $35(222)$ \\
\hline
\end{tabular}

${ }^{a}$ Twenty-five percent of total embryos $(n)$ were expected to be slit mutant; the percentage of slit mutant embryos hatching as L1 was recorded.

${ }^{b}$ Alleles of slit were classified into three groups on the basis of their phenotype, as described in Results.

${ }^{c}$ The percentage of total segments scored $(n)$ containing midline crossovers in embryos heterozygous for both robo and respective alleles of slit.

tracts wander close to the midline (Fig. $1 F, G)$, whereas alleles with a mild midline guidance phenotype have midline deviations in the most medial fasciclin II fascicles and retain an intact lateral fascicle (Fig. 1H,I).

Very few severe or moderate slit mutant embryos hatch; however, up to $17 \%$ of embryos mutant for a mild allele of slit emerge as larvae (Table 1). These larvae are sluggish and uncoordinated and very slow to grow. Most are unable to feed and die in the first larval instar; a very low number pupate or produce adults (Table 1). Mutant larvae have a midline guidance phenotype comparable to the mild embryonic phenotype, characterized by repeated midline crossing of medial fascicles and an unaffected lateral fascicle (Fig. 1, compare $K$ with $J$, wild type).

Our further characterization of slit mutations focuses on representative alleles for severe $\left(s l i^{2}\right)$ (Fig. $\left.2 A\right)$ moderate $\left(s l i^{1912}\right)$ (Fig. 2B), and mild ( $s l i^{2990}$ ) (Fig. 2C) perturbation of midline guidance. Commissure morphology was assessed with BP102 antibody, which labels most CNS axons. Embryos mutant for severe sli alleles have complete medial collapse of the commissures (Fig. $2 A$; compare with wild type in Fig. $6 A$ ). Most axons in embryos mutant for a moderate allele approach the midline, but medial axons are not compressed into a medial fascicle (Fig. 2B). Separation of anterior and posterior commissures is seen in embryos mutant for a weak allele of slit (Fig. 2C).

\section{Interaction with robo}

slit has a dosage-dependent interaction with robo (Kidd et al., 1999). Embryos with a single copy of each gene make occasional midline guidance errors. If the midline guidance phenotype of slit hypomorphs results from reduced signaling to Robo, then we would expect a strong correlation between the severity of the allele of slit and the degree of genetic interaction with the null allele robo ${ }^{1}$. We found that embryos doubly heterozygous for robo ${ }^{1}$ and a mild allele of slit generally had fewer segments with midline guidance errors (Table 1). Nevertheless, more axons can deviate toward the midline in a robo heterozygote with a moderate or mild slit allele (Fig. $2 E, F$ ) than with a severe allele (Fig. 2D). A moderate allele with a strong interaction with robo ${ }^{1}$, such as slit ${ }^{1912}$, may generate Slit protein, which binds to but is a poor activator of the Robo receptor.

\section{Midline guidance of ventral muscles}

slit function also contributes to the positioning of ventral muscle insertions (Battye et al., 1999; Kidd et al., 1999). Normally the ventral oblique muscles are repelled from the midline above the nervous system and insert into the ectoderm underneath the lateral edges of the nerve cord. We found a consistent relationship between the numbers of ventral oblique muscles remaining on the dorsal surface of the nervous system and the class of slit mutant allele. In embryos homozygous for the severe allele $s l^{2}$, an average of 6.8 muscle fibers per segment overlie the nerve cord $(n=11$ segments). In embryos homozygous for a moderate allele, $s l i^{1912}$, 2.9 fibers cross the midline $(n=9)$, whereas $0.6(n=10)$ were found in the hypomorph $s l i^{532}$.

\section{Coding changes in slit mutations}

Nine alleles of slit characterized here were generated by EMS mutagenesis and likely represent point mutations in the slit locus. A sequence change in a region of the Slit protein required for repellent signaling should generate a strong midline guidance phenotype. We generated a map of the intron-exon boundaries of genomic slit based on PCR amplification of gDNA using primers generated from the cDNA sequence. Our map of 19 exons was in agreement with the subsequently released genomic sequence from the Berkeley Drosophila Genome Project (AC005556).

We have sequenced each exon of each EMS allele of slit amplified from gDNA. We identified 19 consistent polymorphisms, one of which results in an alternate amino acid (see Materials and Methods). Allele-specific coding changes were identified for seven EMS alleles (Fig. 3). Four coding changes were identified in the leucine-rich regions and three in the EGF domain. Severe and moderate mutations include a premature stop codon in the last LRR $\left(s l i^{\mathrm{GA} 20}\right)$ and amino acid changes in

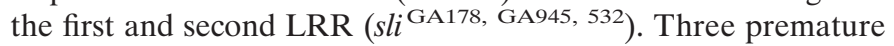
stops in the EGF repeats resulted in either severe $\left(s l i^{2,1912}\right)$ or 


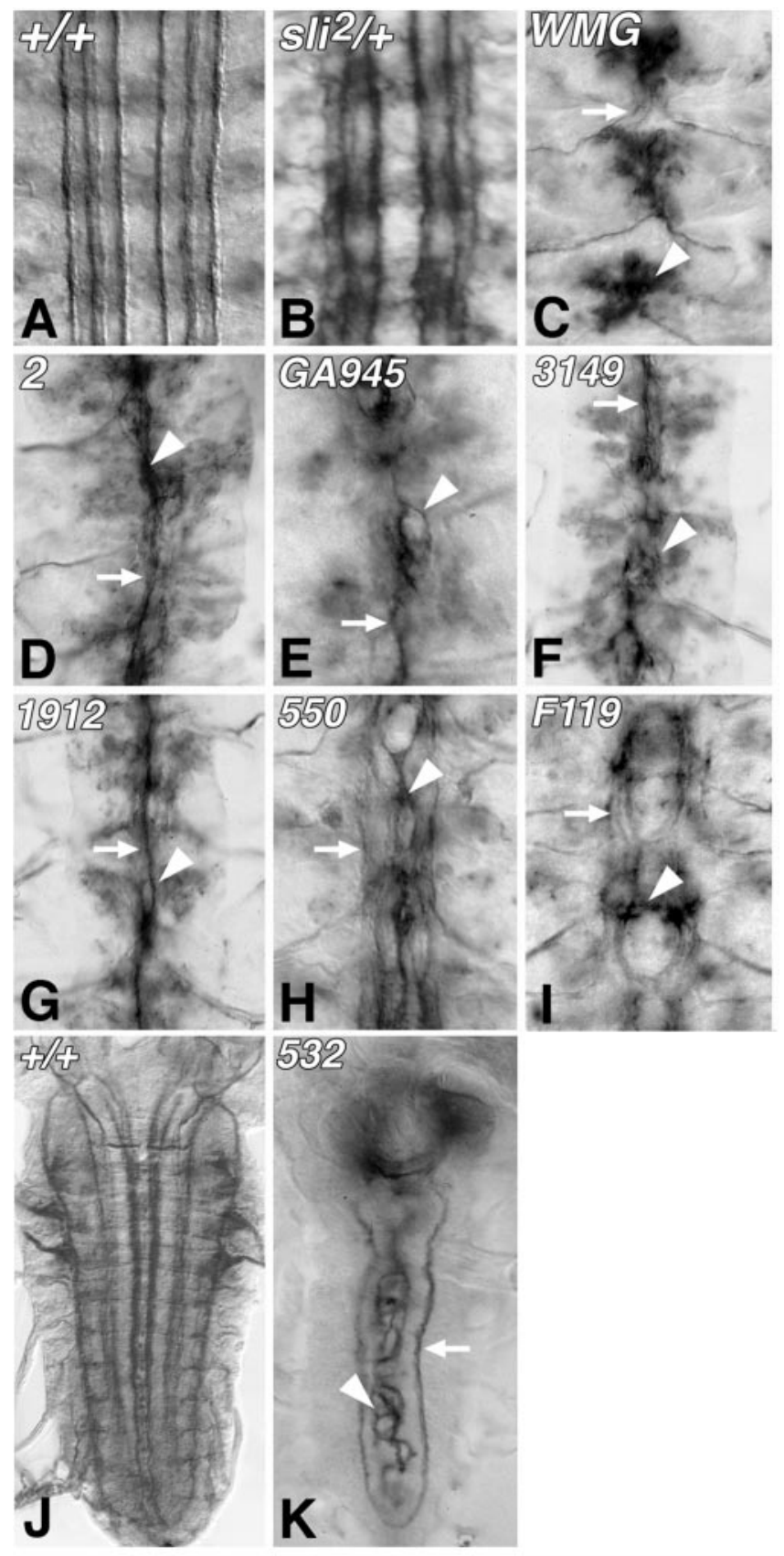

Figure 1. Midline guidance phenotypes of slit nulls and hypomorphs. In wild-type embryos $(A)$, Fasciclin II labels a bilateral set of three fascicles that run symmetrically along the length of the nerve cord but do not cross the midline or each other. A nerve cord heterozygous for $\operatorname{slit}^{2}(B)$ has slight deviations in each longitudinal fascicle, but individual fascicles remain separated. Fasciclin II labeling axons fuse at the midline in embryos deficient for slit $(C)[D f(2 R) W M G]$. Slit alleles with severe $(D$, $E)$, moderate $(F, G)$, and mild $(H, I)$ phenotypes are shown. Alleles that do not make immunodetectable Slit, slit $^{2}(D)$, and slit ${ }^{\mathrm{GA} 945}(E)$ cause a complete fusion of longitudinal axons at the midline. Embryos of moderate alleles of slit that generate detectable protein have longitudinal fascicles that crisscross the midline (arrowhead) and have thicker intersegmental connections [slit ${ }^{3149}(F)$, slit ${ }^{1912}(G)$ ]. Longitudinal axons in the moderate phenotypes still regularly fuse with adjacent fascicles. slit alleles that produced mild phenotypes included $s_{i t}{ }^{550}(H)$ and $s_{i t}{ }^{\mathrm{F} 119}$ (I). Mild slit phenotypes have a larger separation between axon fascicles, and contralateral projections across the midline are restricted to the two most medial fascicles. Anterior is at top in these frontal views of stage 16 nerve cords. Numerous Fasciclin II-labeling longitudinal fascicles are found in a wild-type third instar larval nerve cord $(J)$. A second instar-

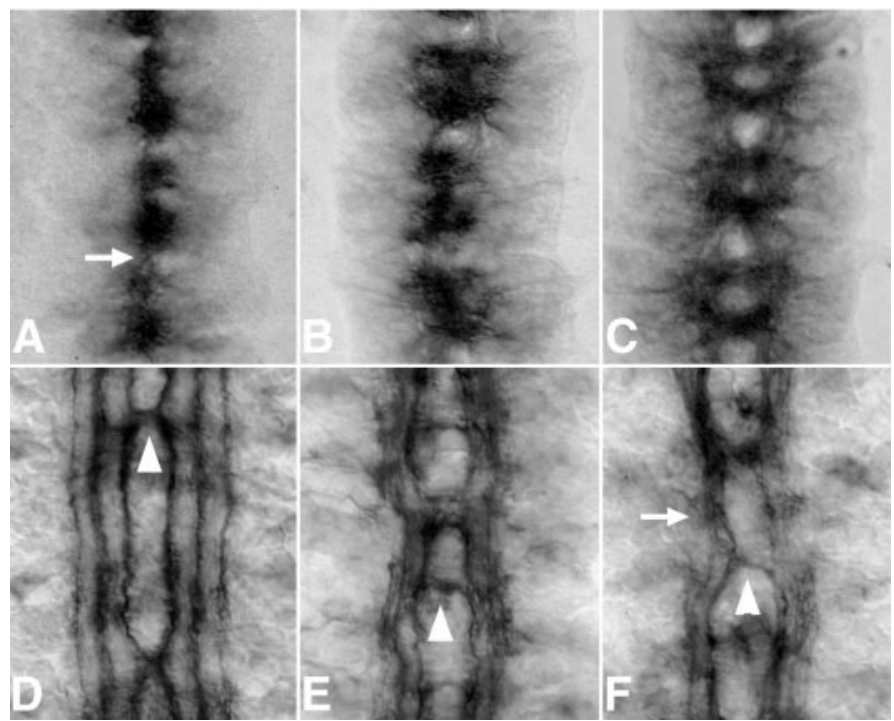

Figure 2. Commissure structure and the genetic interaction of slit alleles with robo ${ }^{1}$. Embryos homozygous for a severe allele of slit $\left(s l{ }^{2}\right)$ lack all commissure structure $(A$, arrow). For comparison, a wild-type axon tract scaffold labeled with BP102 has a ladder-like morphology (Fig. 5A). Less midline compression of axons and more intersegmental axon projections are seen with moderate $\left[s l i{ }^{1912}(B)\right]$ and mild $\left[s l i{ }^{2990}(C)\right]$ alleles. Separation of commissures (arrow) seen in mild slit mutants is accompanied by greater widening of the ventral nerve cord, resembling a robo-like phenotype. The phenotypic interaction with robo ${ }^{1}$ is contrasted with the same alleles. In embryos heterozygous for robo ${ }^{1}$ and slit ${ }^{2}$, deviations are observed in the most medial longitudinal fascicles within a subset of segments $(D$, arrowhead). The remaining fascicles are typical of embryos heterozygous for either slit ${ }^{2}$ or robo ${ }^{1}$. The interactions between robo ${ }^{1}$ and moderate slit alleles [slit $\left.{ }^{1912}(E)\right]$ have prominent crossovers (arrowhead). More lateral fascicles are less organized (arrow). A similar pattern is seen in the slit ${ }^{2990} /$ robo $^{1}$ transheterozygotes $(F)$, including the fusion of lateral fascicles (arrow).

moderate $\left(s l i^{550}\right)$ phenotypes. No sequence changes were revealed in two alleles of slit $\left(s l i^{3149,2990}\right)$, both of which make immunodetectable protein. Noncoding changes may have altered levels of transcription or the regulation of splicing.

\section{Structural requirements for repellent signaling by slit}

Characterized slit mutations alter the sequence of the LRR, truncate the protein, or in the instance of insertional alleles, lower the levels of immunodetected protein (Rothberg et al., 1990). These data do not reveal a contribution to repellent signaling by the EGF, G-, or cysteine domains of Slit. To explore the function of these domains, we sought to identify the repellent signaling potential of slit transgenes that encode forms of Slit with internal deletions.

Drosophila were transformed with three slit transgenes with different internal deletions (Fig. 4), regulated with the binary GAL4-UAS system (Brand and Perrimon, 1993). Using the slit promoter as the GAL4 driver in a homozygous slit ${ }^{2}$ mutant background, we assessed the potential of each transgene to rescue the midline axon guidance phenotype. A complete slit transgene partially rescues a slit mutant, restoring commissural separation,

slit $^{532}$ hypomorph of the same age has disorganized medial fascicles that re-cross the midline ( $K$, arrowhead). The most lateral tract is normal $(K$, arrow). In this and successive Figures, arrows identify intersegmental segments of the longitudinal fascicles, and arrowheads indicate midline guidance errors. 


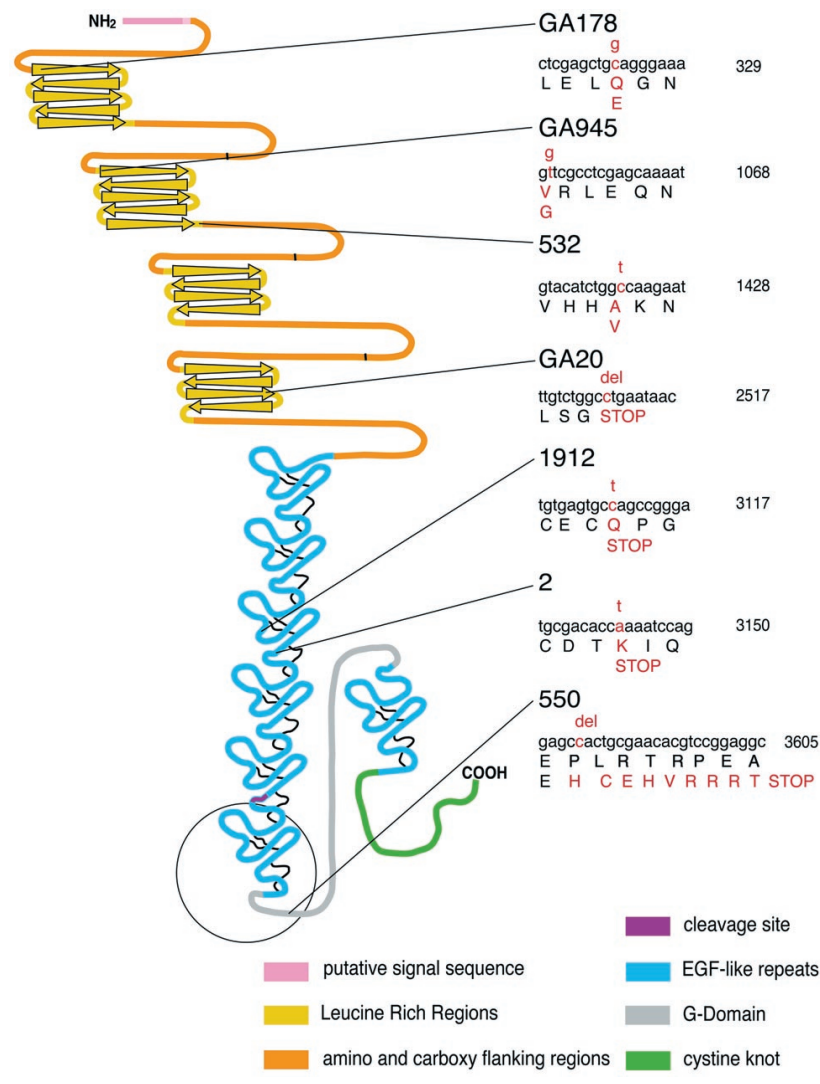

Figure 3. Coding changes of seven slit alleles. Unique changes in the nucleotide and amino acid sequence of slit alleles (right) are mapped to the structural domain of the Slit protein (left) following the protein motif conventions introduced by Rothberg et al. (1990).

although some interruptions in the intersegmental connections persist (Fig. 5B).

Nearly all of the LRR of slit was deleted in Slit[$\Delta \mathrm{L} 1-\mathrm{L} 4]$. This transgene had no effect on the midline axon fusion phenotype of slit $^{2}$ (Fig. 5C). Transgenes with smaller internal deletions in the LRR generated identical results (data not shown). In contrast, removal of the first six EGF repeats (Fig. 5D) or the G-domain and seventh EGF repeat (Fig. $5 E$ ) resulted in restoration of the midline guidance phenotype. This pattern was consistent for three or more independent insertions of each rescue transposon. The deletion construct removing the EGF motifs was not detectable with Slit antibody; however, all transgene products were detectable, at similar levels of imunolabeling intensity, with anti-HA antibody (data not shown). Viability was not restored in any of our "rescue" experiments, suggesting that slit expression elsewhere in the embryo is required for survival.

\section{Ectopic expression of truncated slit transgenes}

Misexpression of $P[U A S$-slit $]$ in embryos disrupts axon tract establishment (Battye et al., 1999; Kidd et al., 1999). To determine

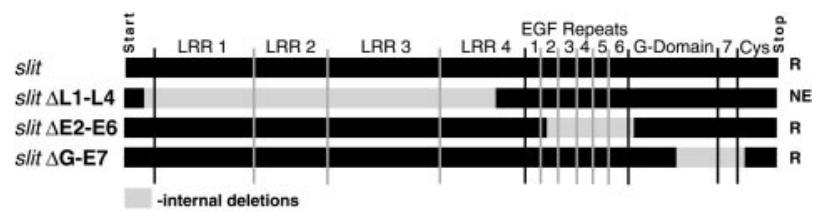

Figure 4. slit transgenes with internal deletions. The regions of slit deleted in three transgenes are lightly shaded. Letters at right indicate repellent activity. $R$, Repellent; $N E$, no effect.

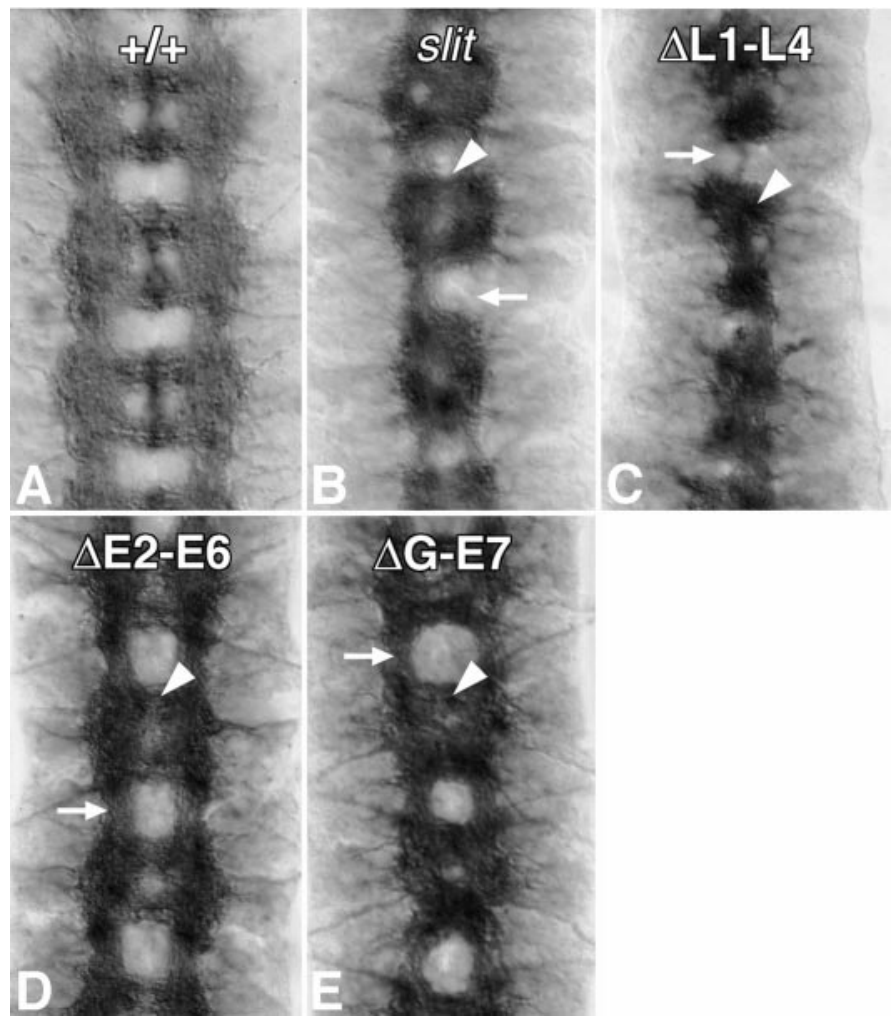

Figure 5. Restoration of slit function using slit transgenes with internal deletions. Possible restoration of axon tract architecture was assessed in Drosophila mutant for slit $^{2}$, also carrying UAS-slit constructs (Fig. 4) expressed under slit1.0-GAL4 regulation. Wild-type expression of Slit in the ventral nerve cord (black) is shown in a BP102-labeled nerve cord $(A)$. The expression of P[UAS-slit complete] was sufficient to partially rescue the slit $^{2}$ phenotype, demonstrated by greater separation of axons from the midline $(B$, arrowhead), although intersegmental connections are not restored $(B$, arrow $)$. A slit transgene lacking the leucine domains of slit $(C)$ $[\mathrm{P}(\mathrm{UAS}-\mathrm{slit} \Delta \mathrm{L} 1-\mathrm{L} 4)]$ did not rescue the slit $^{2}$ phenotype. Midline fusion was not suppressed (arrowhead). A slit transgene lacking five EGF repeats, $\mathrm{P}[\mathrm{UAS}-$ slit $\Delta \mathrm{E} 2-\mathrm{E} 6]$, was able to restore the midline scaffold to near normal conditions, including significant restoration of longitudinal tracts (D). Removal of only the G-domain and final EGF, P[UAS-slitsG-E7] $(E)$, rescued the $s_{l i t}{ }^{2}$ phenotype to a degree similar to slit lacking five EGF repeats (P[UAS-slit $\Delta \mathrm{E} 2-\mathrm{E} 6])(D)$.

which forms of Slit with internal deletions retain the capacity to disrupt axon tract establishment, we misexpressed the slit transgenes in a wild-type pattern (with $P[$ sli1.0-GAL4]), in some neurons and the ectoderm at the segment boundary (with $P[$ engrailed-GAL4]), and in all neurons (with $P[$ elav-GAL4]). The patterns of misexpression are shown in Figure $6, A, F$, and $K$, respectively.

Expression of full-length slit resulted in disruption of intersegmental connections and displacement of axon tracts away from the source of Slit (Fig. 6B,G,L). Expression of slit lacking the LRR domain had no effect on axon tract organization (Fig. $6 C, H, M)$. However, expression of slit lacking the EGF motifs (Fig. $6 D, I, N$ ) or lacking the G-domain (Fig. $6 E, J, O$ ) resulted in significant disruption of axon tract organization. Longitudinal tracts had numerous breaks and lacked uniform bundling. Commissures were lacking when transgenes were expressed in the midline (Fig. 6D,E). Axon tract perturbations were similar for all functional transgenes when expressed in all neurons (Fig. $6 L, N, O)$. 


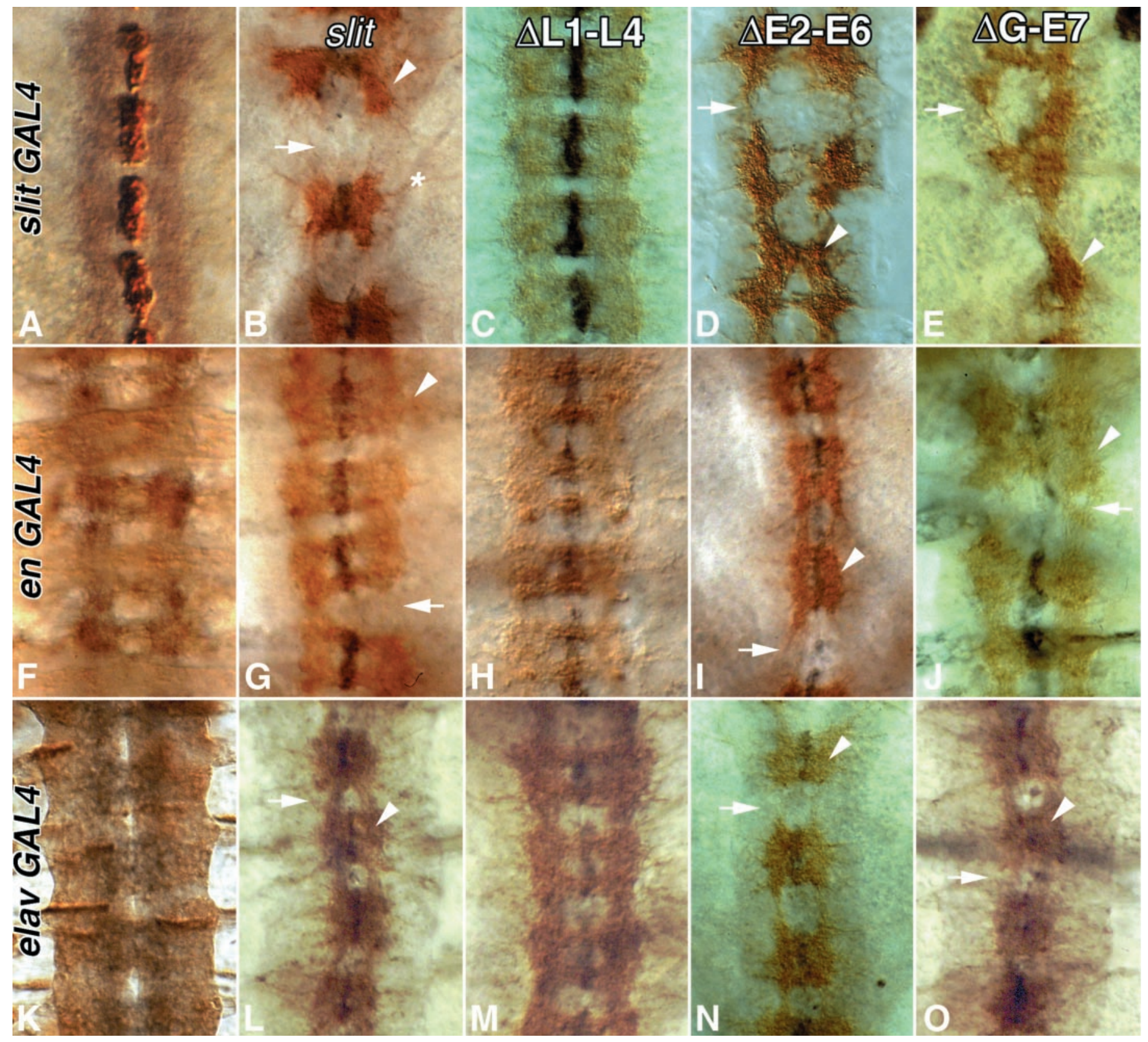

Figure 6. Ectopic expression of slit transgenes with internal deletions. slit transgenes (Fig. 4) were expressed in three different patterns using specific $\mathrm{P}[G A L 4]$ drivers in a wild-type background. $\mathrm{P}[$ slit1.0-GAL4] $(A-E)$ drives expression in the $\mathrm{MG}$, $\mathrm{P}[$ eng-GAL4] $(F-J)$ drives ectopic expression at the segmental boundary, and $\mathrm{P}[$ elav-GAL4] $(K-O)$ directs expression in all neurons. The pattern of ectopic expression is shown in $A, F$, and $K$, where each $\mathrm{P}[G A L 4]$ driver was crossed to P[UAS-tau-LacZ] and embryos were labeled with BP102 (black) and $\alpha-\beta$-gal (brown). In the remaining panels, BP102 (brown) and Slit immunolabeling (black) are visualized. Expression of the complete slit cDNA $(B, G, L)$ disrupted both longitudinal (arrows) and commissural tracts. Some axons misproject laterally $(B$, asterisk). Ectopic expression of P[UAS-slit complete] in all neurons $(L)$ displaced axon tracts toward the midline. Expression of a slit transgene that lacks the leucine domains $(C, H, M)$ did not significantly alter axon tract organization. Expression of a slit transgene lacking EGF repeats 2 through 6 (P[UAS-slit $\Delta$ E2-E6]) significantly altered axon tract organization $(D, I, N)$. Intersegmental expression of P[UAS-slitsE2-E6] resulted in a displacement of axons toward the midline (I, arrowhead) and breaks in the longitudinal tracts (arrow). Expression of P[UAS-slit $\Delta \mathrm{E} 2-\mathrm{E} 6]$ in all neurons also eliminates most intersegmental axons ( $N$, arrow). This construct was not detected by Slit antibody, and only native Slit labeling is seen. Expression of a slit transgene lacking the G-domain and the seventh EGF repeat $(E, J, O)$ results in effects similar to expression of the entire construct. Intersegmental expression of P[UAS-slit $\Delta \mathrm{G}-\mathrm{E} 7]$ resulted in a number of breaks in longitudinal fascicles $(J$, arrow $)$ and poorly defined anterior and posterior commissures $(J$, arrowhead). Expression of P[UAS-slit $\Delta \mathrm{G}-\mathrm{E} 7]$ in all neurons $(O)$ has displaced axons toward the midline (arrowhead).

\section{Structural requirements for Robo binding}

Sequence data from slit mutants and the domain requirements for transgene rescue of slit mutations both suggest that repellent signaling requires the LRR. If repellent signals are transduced by Robo receptors, then we would anticipate that the LRR is re- quired to bind to Robo. We translated the slit transgenes in vitro, incorporating a biotin label, and then bound them to an avidin Agarose column. The column was then incubated with in vitro translated robo (vector provided by K. Bland and C. Goodman). All slit transgenes generated proteins of the predicted size, de- 


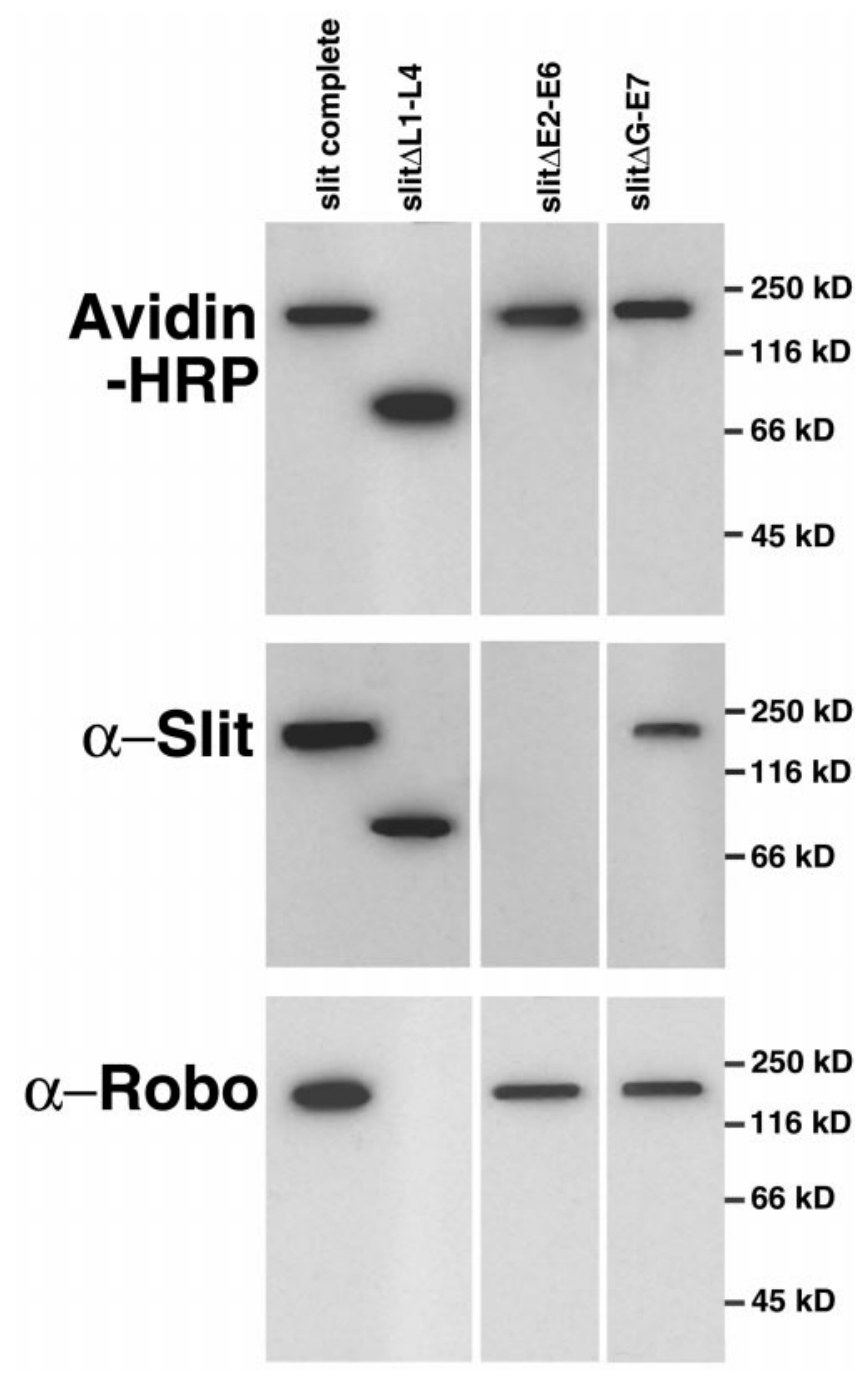

Figure 7. In vitro binding of truncated Slit to Robo. Slit transgenes, both complete and containing internal deletions (Fig. 4), were in vitro translated incorporating biotin-labeled lysine and then bound to avidin Agarose columns. In vitro translated Robo incubated with each column, washed, and eluted with n-biotin. Thirty percent of the extraction from each column was immunoblotted as indicated. Avidin-HRP visualized with chemoluminescence shows that each of the Slit constructs bound and was then eluted from the avidin columns. The Slit antibody detects all constructs except those that delete the EGF2-EGF6 region, to which the antibody binds. The immunoblot for Robo indicates that Slit complete, Slit $\Delta \mathrm{E} 2-\mathrm{E} 6$, and Slit $\Delta \mathrm{G}$ are capable of binding Robo; however, Slit protein with internal deletions of any or all of the LRR fail to demonstrate any detectable robo antigen.

tected with either avidin-HRP or antibody to Slit. Robo was retained only with full-length Slit and Slit lacking either the first six EGF repeats or the G-domain and the last EGF repeat (Fig. 7).

\section{DISCUSSION}

We have combined an analysis of mutations of slit with a functional assay of slit transgenes to determine the structural requirements for repellent signaling by slit. Sequence changes that reduce repellent signaling either shorten the protein or modify the structure of the LRR. Similarly, slit transgenes containing a complete LRR restore axon repellent signals in slit mutants, whereas transgenes that lack a complete LRR generated no repellent signals. Finally, we demonstrated that slit transgenes that retain the re- pellent function produce proteins that bind to Robo, and conversely, Robo did not bind truncated forms of Slit that lack repellent effects.

\section{Dosage-dependent signaling by slit mutants}

The 13 alleles of slit examined here cause a number of midline guidance errors in axons and ventral muscles. Muscle phenotypes range from midline crossovers of all ventral oblique muscles to midline crossing of a few muscle cells per embryo. Axon phenotypes vary from complete midline fusion of all CNS axons to midline crossings of only the most medial axons. Lateral axon tracts are relatively unaffected in mild EMS alleles and P-element insertion alleles of slit. All three P-element insertions have been mapped 10-100 bp upstream of the transcription initiation site and apparently reduce the level of Slit protein produced (Rothberg et al., 1990). It is possible that medial axons, being closer to the midline source of secreted Slit, have a higher threshold to respond to Slit and are thus more sensitive to reduced protein levels in mild alleles. Guidance and lateral positioning of the more lateral axon tracts are regulated by the Robo2 and Robo3 receptors, which may respond at a lower threshold to Slit (Rajagopalan et al., 2000; Simpson et al., 2000a).

\section{Sequence changes in slit mutants}

Three mutations that result in single amino acid changes map to the LRR domain. Point mutations in the $\beta$-sheet of LRR 1 and 2 $\left(s l i^{\mathrm{GA} 178}, s l i^{\mathrm{GA} 945}\right)$ generate a severe phenotype. The sensitivity of slit phenotype to these conservative coding changes suggests a critical requirement of the LRR domain in repellent signaling. All other sequence changes result in truncated proteins with variable portions of the EGF domain preserved. Truncated proteins lacked the epitope recognized by our antibody; therefore, it was not possible to assess their stability or distribution.

Drosophila Slit is likely proteolytically cleaved at the beginning of the sixth EGF repeat (Brose et al., 1999). Slit synthesized in $s i^{550}$ mutants has an incomplete sixth EGF repeat and an altered $\mathrm{C}$-terminal sequence. If the altered $\mathrm{C}$-terminal sequence does not destabilize the protein, we would anticipate that $s l i^{550}$ could act as a hypermorph, signaling in a manner comparable to the $\mathrm{N}$-terminal portion of endogenously cleaved Slit. slit $^{550}$ had the most variable penetrance of all the alleles examined. Nevertheless, the axon guidance phenotype, viability, and robo interaction suggest that sli ${ }^{550}$ is a hypomorph.

Although the G- and cysteine domains of slit represent onefourth of the coding region, no sequence changes map to this region. It is possible that many point mutations in this region are not lethal and would not have been isolated by mutagenesis.

\section{The leucine-rich repeat is required for repellent signaling}

To learn more about the structural requirements for repellent signaling by Slit, we attempted rescue of slit mutants with midline expression of slit transgenes lacking internal sequences. A slit transgene lacking the LRR failed to restore midline guidance and failed to generate effects after ectopic expression. Furthermore, in vitro translated Slit, which lacks a full LRR, did not bind to Robo, the repellent receptor. Point mutations encoding single amino acid changes in the LRR also greatly reduced repellent signaling. These data indicate that the LRR of Slit is required for receptor binding and repellent signaling.

Slit is the first protein for which receptor binding and signaling have demonstrated a requirement for the LRR. The LRR defines a superfamily of proteoglycans of the ECM having tandem re- 
peats of $x x I / V / L x x x x F / P / L x x L / P x x L x x L / I x L x x N x I / L$, where $x$ is any amino acid (for review, see Matsushima et al., 2000). The best-characterized members are decorin and biglycan (for review, see Hocking et al., 1998). A Drosophila cell surface receptor (Toll), a GPI-linked proteoglycan (Connectin), and transmembrane Chaoptin also contain LRR (Rothberg and ArtavanisTsakonas, 1992). LRR-containing proteins of the ECM are implicated in binding of collagen [fibromodulin, decorin, lumican, and biglycan (Hedbom and Heinegard, 1993), laminin (biglycan), and fibronectin [decorin and biglycan (Hocking et al., 1998)]. Biglycan, decorin, and fibromodulin bind TGF- $\beta$ (Hildebrand et al., 1994) and may act as a tumor suppresser (Santra et al., 1997).

Fly LRR-containing proteins are involved in cell adhesion (Keith and Gay, 1990). Connectin is required for homophilic adhesion during motoneuron pathfinding and target recognition in Drosophila (Nose et al., 1997). Toll promotes homophilic adhesion (Keith and Gay, 1990) but also appears to inhibit formation of neuromuscular junctions on Toll-expressing muscle (Rose et al., 1997). Chaoptin is required for fasciculation of photoreceptor axons (Van Vactor et al., 1988). Repellent signaling by Toll and Connectin has been suggested and later discounted (Nose et al., 1994; Rose et al., 1997). The possibility of common functions of LRRs deserves reexamination.

\section{Other structural domains of slit}

It has been suggested that full-length Slit associates with the cell surface and that proteolytic cleavage at the start of the sixth EGF repeat generates two fragments (Brose et al., 1999). Three independent lines of evidence considered here identify a requirement for the LRR, in the N-terminal proteolytic fragment, for Robo binding and repellent signaling. Deletion of the EGF or G-domain did not reduce repellent signaling. These transgenes were more potent than full-length Slit in restoring midline axon guidance in slit mutants. This may be because these truncated proteins were more stable or were expressed at higher levels. Our epitope tag labeling of the transgene products verified the presence of all transgene products but could not resolve relative levels of expression. An alternative interpretation is that the EGF or G-domain may have a regulatory influence on repellent signaling by the LRR, perhaps in establishing the gradient of repellent signal by retention on the MG cell surface. Functions of the EGF and G-domains deserve further investigation.

Robo binding did not require an intact EGF, G-, or cysteine domain. Internal deletion of the EGF domains also removed the putative cleavage site in EGF repeat 6; nevertheless, this protein still signals as a repellent. Therefore, it is likely that uncleaved Slit has repellent signaling function.

Protein interactions of the EGF, G-, and cysteine domains await analysis. They may play a role in binding laminin and Netrin, which bind vetebrate Slit (Brose et al., 1999). The EGF repeats of Slit are very similar to the non-calcium-binding repeats of Notch and Delta. Notch, Delta, Slit, laminin, and sea urchin fibropellin share a PGYTG motif within the EGF region. The EGF domains are implicated in specific protein recognition events, for instance, the recognition of Notch by Delta (Lieber et al., 1992). The laminin EGF domain can promote neurite extension and modulate attractive signaling by netrin (Hopker et al., 1999).

The G-domain of laminin (also termed the ALPS motif) (Rothberg et al., 1990) is also found in Slit, neurexin, agrin, and perlecan, juxtaposed with EGF repeats. All members of this family participate in morphogenetic activities in the ECM. Lami- nin, neurexin, and agrin are also implicated in cell signaling during cell differentiation. Laminin binding to syndecan and integrin requires the G-domain (Hoffman et al., 1998; Kadoya et al., 1998). The G-domains of agrin promote postsynaptic differentiation of the neuromuscular synapse. Removal of the adjacent EGF repeats enhances this activity (Cornish et al., 1999). Our study did not reveal an essential role for the G-domain in repellent signaling by Slit. The role of this domain in Slit may be revealed when its binding partner is identified.

The $\mathrm{C}$ terminus of vertebrate Slit contains a cysteine knot (Brose et al., 1999), also found in growth factors that dimerize (McDonald and Hendrickson, 1993). The C terminal of Drosophila Slit, also cysteine rich, does not have appropriately spaced cysteines to form looped intermolecular disulfate bonds. Our internal deletion constructs that retained repellent effects did not remove the cysteine domain; therefore, we cannot exclude a contribution from this domain in Slit signaling.

In contrast to the other domains of the Slit protein, only the LRR domain is required in slit transgenes to restore midline guidance. Furthermore, translated Slit that lacks a full LRR did not bind to Robo, the repellent receptor. These data and the mutant sequence data indicate that LRR of Slit is required for, although not necessarily sufficient for, receptor binding and repellent signaling. This is the first demonstration that the LRR motif can function as a ligand for signal transduction.

\section{REFERENCES}

Battye R, Stevens A, Jacobs JR (1999) Axon repulsion from the midline of the Drosophila CNS requires slit function. Development 126:2475-2481.

Brand AH, Perrimon N (1993) Targeted gene expression as a means of altering cell fates and generating dominant phenotypes. Development 118:401-415.

Brose K, Bland KS, Wang K-H, Arnott D, Henzel W, Goodman CS, Tessier-Lavigne M, Kidd T (1999) Evolutionary conservation of the repulsive guidance function of Slit proteins and their interactions with robo receptors. Cell 96:795-806.

Cornish T, Chi J, Johnson S, Lu Y, Campanelli JT (1999) Globular domains of agrin are functional units that collaborate to induce acetylcholine receptor clustering. J Cell Sci 112:1213-1223.

Gay NJ, Packman LC, Weldon MA, Barna JC (1991) A leucine-rich repeat peptide derived from the Drosophila Toll receptor forms extended filaments with a beta-sheet structure. FEBS Lett 291:87-91.

Hedbom E, Heinegard D (1993) Binding of fibromodulin and decorin to separate sites on fibrillar collagens. J Biol Chem 268:27307-27312.

Hildebrand A, Romaris M, Rasmussen LM, Heinegard D, Twardzik DR, Border WA, Ruoslahti E (1994) Interaction of the small interstitial proteoglycans biglycan, decorin and fibromodulin with transforming growth factor beta. Biochem J 302:527-534.

Hocking AM, Shinomura T, McQuillan DJ (1998) Leucine-rich repeat glycoproteins of the extracellular matrix. Matrix Biol 17:1-19.

Hoffman MP, Nomizu, M, Roque E, Lee S, Jung DW, Yamada Y, Kleinman HK (1998) Laminin-1 and laminin-2 G-domain synthetic peptides bind syndecan-1 and are involved in acinar formation of a human submandibular gland cell line. J Biol Chem [Erratum (1999) 274:12950] 273:28633-28641.

Hopker VH, Shewan D, Tessier-Lavigne M, Poo M, Holt C (1999) Growth-cone attraction to netrin-1 is converted to repulsion by laminin-1. Nature 401:69-73.

Jacobs JR (2000) The midline glia of Drosophila: a molecular genetic model for the developmental functions of glia. Prog Neurobiol 62:475-508.

Kadoya Y, Nomizu M, Sorokin LM, Yamashina S, Yamada Y (1998) Laminin alpha1 chain $G$ domain peptide, RKRLQVQLSIRT, inhibits epithelial branching morphogenesis of cultured embryonic mouse submandibular gland. Dev Dyn 212:394-402.

Keith FJ, Gay NJ (1990) The Drosophila membrane receptor Toll can function to promote cellular adhesion. EMBO J 9:4299-4306.

Kennedy TE, Serafini T, de la Torre JR, Tessier-Lavigne M (1994) Netrins are diff usible chemotropic factors for commissural axons in the embryonic spinal cord. Cell 78:425-435.

Kidd T, Bland K, Goodman CS (1999) Slit is the midline repellent for the Robo receptor in Drosophila. Cell 96:785-794.

Li HS, Chen JH, Wu W, Fagaly T, Zhou L, Yuan W, Dupuis S, Jiang ZH, Nash W, Gick C, Ornitz DM, Wu JY, Rao Y (1999) Vertebrate slit, a 
secreted ligand for the transmembrane protein roundabout, is a repellent for olfactory bulb axons. Cell 96:807-818.

Lieber T, Wesley CS, Alcamo E, Hassel B, Krane JF, Campos-Ortega JA, Young MW (1992) Single amino acid substitutions in EGF-like elements of Notch and Delta modify Drosophila development and affect cell adhesion in vitro. Neuron 9:847-859.

Lin DM, Goodman CS (1994) Ectopic and increased expression of Fasciclin II alters motoneuron growth cone guidance. Neuron 13:507-523.

Matsushima N, Ohyanagi T, Tanaka T, Kretsinger RH (2000) Supermotifs and evolution of tandem leucine-rich repeats within the small proteoglycans-biglycan, decorin, lumican, fibromodulin, PRELP, keratocan, osteoadherin, epiphycan, and osteoglycin. Proteins 38:210-225.

McDonald NQ, Hendrickson WA (1993) A structural superfamily of growth factors containing a cystine knot motif. Cell 73:421-424.

Murray MJ, Whitington PM (1999) Effects of roundabout on growth cone dynamics, filopodial length, and growth cone morphology at the midline and throughout the neuropile. J Neurosci 19:7901-7912.

Nose A, Mahajan VB, Goodman CS (1992) Connectin: a homophilic cell adhesion molecule expressed on a subset of muscles and the motoneurons that innervate them in Drosophila. Cell 70:553-567.

Nose A, Takeichi M, Goodman CS (1994) Ectopic expression of connectin reveals a repulsive function during growth cone guidance and synapse formation. Neuron 13:525-539.

Nose A, Umeda T, Takeichi M (1997) Neuromuscular target recognition by a homophilic interaction of connectin cell adhesion molecules in Drosophila. Development 124:1433-1441.

Nüsslein-Volhard C, Wieschaus E, Kluding H (1984) Mutations affecting the pattern of the larval cuticle in Drosophila melanogaster I. Zygotic loci on the second chromosome. Roux's Arch Dev Biol 193:267-282.

Patel N (1994) Imaging neuronal subsets and other cell types in wholemount Drosophila embryos and larvae using antibody probes. Methods Cell Biol 44:445-505.

Rajagopalan S, Vivancos V, Nicolas E, Dickson BJ (2000) Selecting a longitudinal pathway: Robo receptors specify the lateral position of axons in the Drosophila CNS. Cell 103:1033-1045.

Rose D, Zhu X, Kose H, Hoang B, Cho J, Chiba A (1997) Toll, a muscle cell surface molecule, locally inhibits synaptic initiation of the RP3 motoneuron growth cone in Drosophila. Development 124:1561-1571.

Rothberg JM, Artavanis-Tsakonas S (1992) Modularity of the slit protein. Characterization of a conserved carboxy-terminal sequence in secreted proteins and a motif implicated in extracellular protein interactions. J Mol Biol 227:367-370.

Rothberg JM, Hartley DA, Walther Z, Artavanis-Tsakonas S (1988) slit: an EGF-homologous locus of D. melanogaster involved in the development of the embryonic central nervous system. Cell 55:1047-1059.

Rothberg JM, Jacobs JR, Goodman CS, Artavanis-Tsakonas S (1990) slit: an extracellular protein necessary for development of midline glia and commissural axon pathways contains both EGF and LRR domains. Genes Dev 4:2169-2187.

Santra M, Mann DM, Mercer EW, Skorski T, Calabretta B, Iozzo RV (1997) Ectopic expression of decorin protein core causes a generalized growth suppression in neoplastic cells of various histogenetic origin and requires endogenous p21, an inhibitor of cyclin-dependent kinases. J Clin Invest 100:149-157.

Seeger M, Tear G, Ferres-Marco D, Goodman CS (1993) Mutations affecting growth cone guidance in Drosophila: genes necessary for guidance toward or away from the midline. Neuron 10:409-426.

Simpson JH, Bland KS, Fetter RD, Goodman CS (2000a) Short-range and long-range guidance by Slit and its Robo receptors: a combinatorial code of Robo receptors controls lateral position. Cell 103:1019-1032.

Simpson JH, Kidd T, Bland KS, Goodman CS (2000b) Short-range and long-range guidance by Slit and its Robo receptors: Robo and Robo2 play distinct roles in midline guidance. Neuron 28:753-766.

Tessier-Lavigne M, Goodman CS (1996) The molecular biology of axon guidance. Science 274:1123-1133.

Van Vactor Jr D, Krantz DE, Reinke R, Zipursky SL (1988) Analysis of mutants in chaoptin, a photoreceptor cell-specific glycoprotein in Drosophila, reveals its role in cellular morphogenesis. Cell 52:281-290.

Verheyen EM, Cooley L (1994) Profilin mutations disrupt multiple actin-dependent processes during Drosophila development. Development 120:717-728.

Wang KH, Brose K, Arnott D, Kidd T, Goodman CS, Henzel W, Tessier-Lavigne M (1999) Biochemical purification of a mammalian slit protein as a positive regulator of sensory axon elongation and branching. Cell 96:771-784.

Yamagata M, Merlie JP, Sanes JR (1994) Interspecific comparisons reveal conserved features of the Drosophila Toll protein. Gene 139:223-228.

Yuan W, Zhou L, Chen JH, Wu JY, Rao Y, Ornitz DM (1999) The mouse SLIT family: secreted ligands for ROBO expressed in patterns that suggest a role in morphogenesis and axon guidance. Dev Biol 212:290-306.

Zhou Y, Stoeckli E, Chen H, Tessier-Lavigne M (2000) Squeezing axons out of the gray matter: a role for Slit and Semaphorin proteins from midline and ventral spinal cord. Cell 102:363-375. 